\title{
Hemodynamic Depression of Early Carotid Revascularization on Ischemic Stroke Patients with Symptomatic Carotid Stenosis
}

\author{
Jun Woo Kim, MD and Youngrok Do, MD \\ Department of Neurology, Daegu Catholic University Medical Center, Catholic University of Daegu School of Medicine, Daegu, Korea
}

Background: Carotid revascularization is frequently complicated by hemodynamic depression (HD), which can increase the risk of stroke or vascular death. However, no studies yet exist describing the correlation between the timing of carotid revascularization and HD. The present study investigated whether carotid revascularization in the early phase of a stroke had an impact on the incidence of HD.

Methods: A retrospective study of consecutive ischemic stroke patients who underwent carotid revascularization was designed to compare the incidence of HD between early and late carotid revascularization. Early carotid revascularization was defined as carotid artery stenting or endarterectomy within two weeks of stroke onset. HD was defined as hypotension or bradycardia within five days of carotid revascularization.

Results: Of 69 carotid revascularizations, HD occurred in 29 (42.0\%). The incidence of HD did not differ significantly between the early and late phase groups (48.0\% vs. $38.6 \%$, respectively, $P=0.449)$. Patients with $\mathrm{HD}$ had undergone more carotid artery stenting than those without HD $(79.3 \%$ vs. 35\%, respectively, $P<0.001)$. Age, sex, hypertension, diabetes, heart disease, antihypertensive medication, and severity of carotid stenosis did not differ between patients with and without HD.

Conclusion: The present research suggests that early carotid revascularization is as safe as late carotid revascularization with regard to HD.

J Neurocrit Care 2017;10(2):92-98

Key words: Endarterectomy, Carotid; Stents; Stroke; Hypotension; Bradycardia; Hemodynamics

\author{
Received June 20, 2017 \\ Revised August 21, 2017 \\ Accepted September 1, 2017 \\ Corresponding Author: \\ Youngrok Do, MD \\ Department of Neurology, Daegu \\ Catholic University Medical Center, \\ Catholic University of Daegu School of \\ Medicine, 33 Duryugongwon-ro 17-gil, \\ Nam-gu, Daegu 42472, Korea \\ Tel: +82-53-650-3064 \\ Fax: +82-53-654-9786 \\ E-mail:dyr4173@cu.ac.kr
}

Copyright $\odot 2017$ The Korean Neurocritical Care Society

\section{INTRODUCTION}

Carotid revascularization is commonly performed in stroke patients with carotid artery stenosis in order to prevent the recurrence of ischemic stroke. ${ }^{1,2}$ Hemodynamic depression (HD) is a common complication that follows carotid revascularization. Although a meta-analysis demonstrated that the incidence of clinical events was not associated with HD, ${ }^{3}$ HD has caused clinical events, such as stroke, myocardial infarction, and mortality. ${ }^{4,5}$ Moreover, $\mathrm{HD}$ is a generally challenging event for clinicians to prevent brain as well as systemic hypoperfusion.

According to the treatment guidelines for carotid stenosis, an early carotid endarterectomy (CEA) is recommended within two weeks of stroke onset for patients who experienced a stable stroke in order to prevent early stroke recurrence. ${ }^{6}$ On the other hand, it is known that the early phase of strokes is hemodynamically unstable. This lack of

(cc) This is an Open Access article distributed under the terms of the Creative Commons Attribution Non-Commercial License (http://creativecommons.org/licenses/by$\mathrm{nc} / 4.0$ ) which permits unrestricted non-commercial use, distribution, and reproduction in any medium, provided the original work is properly cited. 
stability leads to the speculation that patients undergoing early carotid revascularization are vulnerable to HD. However, there is no available data on the incidence of HD in accordance with the timing of carotid revascularization. The present study aimed to investigate the incidence of HD between early and late carotid revascularization in patients with symptomatic carotid stenosis.

\section{METHODS}

\section{Study design and patients}

In the current single-center, retrospective study, we evaluated the medical records of consecutive patients who underwent carotid revascularization between January 2011 and December 2014. The inclusion criteria were as follows: (1) patients who were 19 years or older in age and (2) patients who underwent CEA or carotid artery stenting (CAS) due to more than $50 \%$ stenosis in the carotid bulb or proximal internal carotid artery. Exclusion criteria were as follows: (1) patients who had asymptomatic carotid stenosis, (2) patients who received simultaneous carotid revascularization with acute endovascular thrombectomy, and (3) patients without regular vital sign records during and after carotid revascularization. Patients who underwent carotid revascularization within and after two weeks following the onset of stroke were assigned to the early and late carotid revascularization groups, respectively. The institutional review board of our medical institution approved the present research. Informed consent was waived due to the retrospective nature of the study.

\section{Clinical and imaging evaluations}

We evaluated the baseline characteristics of all patients, including age, sex, timing of carotid revascularization from stroke onset, past history of hypertension, diabetes, hyperlipidemia, heart failure, coronary artery disease, use of anti-hypertensive agents and statin, the degree of carotid artery stenosis, high level carotid stenosis, and opposite carotid occlusion. Hyperlipidemia was defined as a past history of hyperlipidemia, total cholesterol of $200 \mathrm{mg} / \mathrm{dL}$ or greater, or low-density lipoprotein cholesterol of $130 \mathrm{mg} /$ $\mathrm{dL}$ or greater on the serum biochemistry. Heart failure was defined as a past history of heart failure or an ejection fraction of less than $30 \%$ on a transthoracic echocardiography in patients presenting with symptoms that were suggestive of heart failure during the initial evaluation for acute cerebral infarction. Coronary artery disease was defined as a history of coronary artery disease or newly detected coronary artery disease at the time of the diagnosis of cerebral infarction. Two neurologists who were blinded to patient information independently measured the degree of carotid artery stenosis, high level carotid stenosis, and opposite carotid occlusion. They were evaluated via magnetic resonance angiography or computed tomography angiography. The degree of carotid artery stenosis was measured according to the North American Symptomatic Carotid Endarterectomy Trial method. When the two neurologists disagreed, a third investigator (neuroradiologist) was consulted to facilitate a consensus.

\section{Procedures protocol}

CEA was performed under general anesthesia by a single cardiovascular and thoracic surgeon. After an incision was made in the neck, the carotid artery was exposed and subsequently clamped to prevent embolization. A transcranial Doppler was applied over the ipsilateral middle cerebral artery, and an intraluminal shunt was employed when the Doppler flow was less than $40 \%$ of the baseline flow. A longitudinal arteriotomy was performed, and the carotid plaque was freed with a spatula and forceps. After meticulous inspection, irrigation, and bleeding control of the endarterectomized surface to remove any residual plaques or debris, the arteriotomy was closed with a bovine pericardial patch and 6-0 prolene. Just before completion of the arterial closure, the carotid clamps were briefly released sequentially and re-clamped to back-bleed and forwardflush the vessel, which was then irrigated and suctioned for any residual debris. If there were no other complications or infections following the procedure, the corresponding patients were discharged after receiving a single dose of clopidogrel. 
CAS was performed by a single neuroradiologist with a consistent technique, as described in previous articles. ${ }^{7}$ In brief, aspirin (100 mg/day) and clopidogrel (75 mg/day) were administered for one week prior to the procedure, and a bolus of heparin $(100 \mathrm{U} / \mathrm{kg})$ was administered intravenously during the procedure. Through femoral access, a 6-F sheath (Shuttle SL Flexor ${ }^{\circledR}$; Cook, Bloomington, IN, USA) was advanced into the common carotid artery. A FilterWire (Boston Scientific, Natick, MA, USA) or EmboShield ${ }^{\circledR}$ (Abbott Vascular Devices, Redwood City, CA, USA) was inserted for cerebral protection. A balloon angioplasty was performed with a 4-6-mm Aviator ${ }^{\text {TM }}$ balloon (Cordis, Miami Lakes, FL, USA) before stent placement. Self-expanding Cordis Precise ${ }^{\circledR}$ Carotid Nitinol Stents (Miami Lakes, FL, USA) were deployed. Postdilation was performed at the discretion of the operator. Atropine was administered immediately before the balloon angioplasty with bradycardia of less than 50 times/minute during the procedure. Aspirin and clopidogrel were maintained for at least one month after the procedure.

All patients underwent either CEA or CAS based upon our judgment. Vascular status, presence of contralateral carotid artery steno-occlusion, concurrent diseases, and severity and progress status of stroke symptoms were considered.

\section{Hemodynamic depression}

HD was defined as a systolic blood pressure of less than $90 \mathrm{mmHg}$ or pulse rate of less than 50 times/minute that occurred at least once within the five days during and following carotid revascularization. HD was classified as either intra-procedural, post-procedural, or HD requiring treatment. HD requiring treatment was defined as a $H D$ required for administration of an intravenous medication to normalize blood pressure or pulse rate. Blood pressure and pulse rate were evaluated in the operating, recovery, and procedure rooms, as well as in the ward. The anesthesiologist monitored blood pressure and pulse rates in 15-minute intervals during CEA. We dismissed the transient decreases in blood pressure or pulse rate due to the general anesthesia that was induced during CEA. A vascular intervention nurse monitored blood pressure and pulse rates in 15-minute intervals during the CAS procedure. Blood pressure and pulse rates were monitored in 1-minute intervals during direct manipulation of the carotid artery. Moreover, blood pressure and pulse rates were post-procedurally monitored in 10-minute intervals for 30 minutes in the recovery room, in 1-hour intervals for over two days in the intensive care or stroke unit, and in 8-hour intervals for the following three days in the ward. Patients with a systolic blood pressure of less than $90 \mathrm{mmHg}$ for 30 minutes received intravenous norepinephrine or dopamine, and administration of an anti-hypertensive agent was discontinued until the HD was corrected.

\section{Statistical analysis}

The baseline clinical and imaging characteristics of patients and incidence of HD were compared between the early and late carotid revascularization groups. Chi-square tests and Fisher's exact tests were used to compare all categorical variables between the two groups. In addition, $t$-tests were used to compare the age and mean time from stroke onset to carotid revascularization between the groups. Risk factor candidates for HD, including early carotid revascularization, were analyzed according to the presence of HD. Moreover, the incidence of HD, intraprocedural $H D$, post-procedural HD, and HD requiring treatment after early carotid revascularization, CEA, and CAS were compared between the early and late procedure groups. Statistical analyses were performed using SPSS for Windows version 18.0 (SPSS Inc., Chicago, IL, USA). A P of less than 0.05 was considered statistically significant.

\section{RESULTS}

Ninety-six carotid revascularizations were performed in 93 patients between January 2011 and December 2014. Among 96 carotid revascularizations, 27 were excluded because of the following: (1) 23 had asymptomatic carotid stenosis and (2) 4 received carotid revascularization and acute endovascular thrombectomy simultaneously. Vital signs for all patients were recorded during and after carotid 
Table 1. Characteristics of patients and hemodynamic depression according to the timing of carotid revascularization

\begin{tabular}{|c|c|c|c|c|}
\hline & All $(n=69)$ & $\begin{array}{l}\text { CR within } 2 \text { weeks } \\
\quad(n=25,36.2 \%)\end{array}$ & $\begin{array}{l}\text { CR after } 2 \text { weeks } \\
(n=44,63.8 \%)\end{array}$ & $P$ value \\
\hline Age (years) & $70.7 \pm 8.4$ & $72.0 \pm 8.2$ & $70.0 \pm 8.4$ & 0.337 \\
\hline Male & $51(73.9)$ & $21(84.0)$ & $30(68.2)$ & 0.253 \\
\hline Hypertension & $49(71.0)$ & $19(76.0)$ & $30(68.2)$ & 0.491 \\
\hline Diabetes mellitus & $21(30.4)$ & $5(20.0)$ & $16(36.4)$ & 0.156 \\
\hline Hyperlipidemia & $19(27.5)$ & $5(20.0)$ & $14(31.8)$ & 0.291 \\
\hline Heart failure & $5(7.2)$ & $1(4.0)$ & $4(9.1)$ & 0.646 \\
\hline Coronary artery disease & $10(14.5)$ & $3(12.0)$ & $7(15.9)$ & 0.737 \\
\hline Carotid stenosis $>70 \%$ & $66(95.7)$ & $24(96.0)$ & $42(95.5)$ & 1.000 \\
\hline High level carotid stenosis & $11(15.9)$ & $5(20.0)$ & $6(13.6)$ & 0.511 \\
\hline Opposite carotid occlusion & $2(2.9)$ & $0(0.0)$ & $2(4.5)$ & 0.531 \\
\hline Carotid artery stenting & $37(53.6)$ & $17(68.0)$ & $20(45.5)$ & 0.071 \\
\hline Time from onset to CR (day) & $28.1 \pm 26.4$ & $10.0 \pm 3.0$ & $38.3 \pm 28.3$ & $<0.001$ \\
\hline Hemodynamic depression & $29(42.0)$ & $12(48.0)$ & $17(38.6)$ & 0.449 \\
\hline
\end{tabular}

$\mathrm{CR}$, carotid revascularization.

Values are presented as mean \pm standard deviation or number (\%); categorical data are provided as frequencies (\%).

revascularization.

Of the 69 enrolled carotid revascularizations, 25 (36.2\%) underwent early carotid revascularization and the mean time from stroke onset to carotid revascularization was 10 days. Twenty-nine patients (42.0\%) presented with HD. The incidence of HD did not differ significantly between the early and late carotid revascularization groups (48\% vs. 38.6\%, $P=0.449$ ) (Table 1). Patients with HD had undergone more CAS than those without HD (79.3\% vs. 35\%, $P<0.001)$. Age, sex, hypertension, diabetes, heart disease, antihypertensive medication, severity of carotid stenosis, high level carotid stenosis, and opposite carotid occlusion did not differ between the patients with and without HD (Table 2). After HD was further subdivided into intra-procedural HD, postprocedural HD, and HD requiring treatment, early carotid revascularization still did not significantly differ between patients with and without each type of HD. When early carotid revascularization was divided into early CEA and CAS, both early CEA and CAS were not associated with HD, intra-procedural $H D$, post-procedural $H D$, or $H D$ requiring treatment (Table 3). Post-stent ballooning was performed in 3 patients (6.8\%) in the early CAS group and 3 patients $(12.0 \%)$ in the late CAS group. The incidences of HD by post-stent ballooning in each group were $2(66.7 \%)$ in the early CAS group and 3 (100\%) in the late CAS group.

\section{DISCUSSION}

The present study investigated whether early carotid revascularization increases HD compared to late carotid revascularization. This study demonstrated that no significant difference in the incidence of HD between early and late carotid revascularization exists, which indicates that early carotid revascularization does not increase HD compared to late carotid revascularization. There may be a possible explanation for this finding. Acute hypertensive response, which is reported in $52 \%$ of stroke patients at the early stroke stage, may play a role. ${ }^{8}$ The acute hypertensive response typically decreases gradually after symptom onset, with a systolic blood pressure decrease of $10 \mathrm{mmHg}$ during 24 hours and a decrease of $20 \mathrm{mmHg}$ during the first 10 days. ${ }^{9,10}$ Therefore, HD may be more compensated for by an acute hypertensive response within 2 weeks rather than after 2 weeks of a stroke. The mechanisms of acute hypertensive response are different from that of $\mathrm{HD}$ after carotid revascularization. Acute hypertensive responses can be caused by a Cushing response to the mass effect of brain 
Table 2. Characteristics of patients according to hemodynamic depression

\begin{tabular}{|c|c|c|c|}
\hline & $\mathrm{HD}(+)(n=29,42.0 \%)$ & HD (-) $(n=40,58.0 \%)$ & $P$ value \\
\hline Age, years & $71.7 \pm 9.0$ & $70.0 \pm 7.9$ & 0.407 \\
\hline Male & $22(75.9)$ & $29(72.5)$ & 0.754 \\
\hline Hypertension & $20(69.0)$ & $29(72.5)$ & 0.749 \\
\hline Diabetes mellitus & $8(27.6)$ & $13(32.5)$ & 0.661 \\
\hline Hyperlipidemia & $11(37.9)$ & $8(20.0)$ & 0.100 \\
\hline Heart failure & $2(6.9)$ & $3(7.5)$ & 1.000 \\
\hline Coronary artery disease & $2(6.9)$ & $8(20.0)$ & 0.174 \\
\hline Anti-hypertensive agents & $10(34.5)$ & $17(42.5)$ & 0.501 \\
\hline ACE inhibitor/ARB & $8(27.6)$ & $13(32.5)$ & 0.661 \\
\hline Beta blocker & $3(10.3)$ & $6(15.0)$ & 0.724 \\
\hline Calcium channel blocker & $5(17.2)$ & $8(20.0)$ & 0.772 \\
\hline Diuretics & $2(6.9)$ & $2(5.0)$ & 1.000 \\
\hline Alpha blocker & $2(6.9)$ & $6(15.0)$ & 0.453 \\
\hline Statin & $24(82.8)$ & $32(80.0)$ & 0.772 \\
\hline High intensity statin & $20(69.0)$ & $25(62.5)$ & 0.578 \\
\hline Carotid stenosis $>70 \%$ & $29(100.0)$ & $37(92.5)$ & 0.258 \\
\hline High level carotid stenosis & $0(0.0)$ & $2(5.0)$ & 0.506 \\
\hline Opposite carotid occlusion & $5(17.2)$ & $6(15.0)$ & 0.802 \\
\hline Carotid artery stenting & $23(79.3)$ & $14(35.0)$ & $<0.001$ \\
\hline Early CR & $12(41.4)$ & $13(32.5)$ & 0.449 \\
\hline
\end{tabular}

$\mathrm{HD}$, hemodynamic depression; ACE, angiotensin converting enzyme; ARB, angiotensin receptor blocker; CR, carotid revascularization. Values are presented as mean \pm standard deviation or number (\%); categorical data are given as frequencies (\%).

Table 3. HD, intra-procedural HD, post-procedural HD, and HD requiring treatment after early carotid revascularizations compared to those after late procedures

\begin{tabular}{lcccc}
\hline & HD & Intra-HD & Post-HD & HD-RT \\
\hline Early CR & 0.449 & 0.414 & 0.714 & 0.532 \\
Early CEA & 0.148 & 0.148 & 1.000 & 0.148 \\
Early CAS & 0.286 & 0.431 & 1.000 & 0.272 \\
\hline
\end{tabular}

$\mathrm{HD}$, hemodynamic depression; Intra-HD, intra-procedural HD; Post-HD, post-procedural HD; HD-RT, HD requiring treatment; $C R$, carotid revascularization; CEA, carotid endarterectomy; CAS, carotid artery stenting.

Values are presented as $P$ value compared to late procedures.

edema, ${ }^{11}$ the shifting of the cerebral autoregulation to a higher level, ${ }^{8}$ neuroendocrine responses with activation of the sympathetic nervous system and the renin-angiotensin axis, $^{12}$ and the lesion of prefrontal and insular cortices leading to disruption of normal autonomic control; hence, an exaggerated sympathetic response results. ${ }^{13,14}$ On the other hand, the manipulation of the carotid bulb increases afferent signals transmitted through Hering's nerve to the glossopharyngeal nerve and nucleus tractus solitaries in the medulla. Secondary efferent signals inhibit the activity of the vasoconstrictor center in the medulla and modify the vagal parasympathetic center, which decrease both systemic vascular resistance and cardiac output that results in the lowering of arterial pressure..$^{15-19}$

In the current study, the following did not affect HD: old age, a past history of heart failure, coronary artery disease, severe stenosis of the carotid artery, high level carotid stenosis, and opposite carotid occlusion. To date, risk factors of HD include old age, heart failure, previous myocardial infarction, absence of chronic obstructive pulmonary dis- 
eases, severe stenosis of the carotid artery involving the carotid bulb, long plaque length, severe calcified plaque, ulceration of the plaque, a higher balloon dilatation pressure on the CAS, and the presence of stenosis on the contralateral side. However, these proposed risk factors are still controversial. ${ }^{20-25}$

There was great variability in the incidence rate of $\mathrm{HD}$ following CEA and CAS, which ranged from $12-40 \%$ and $5-76 \%$, respectively. It is generally known that the incidence of HD is higher in CAS compared to CEA., ${ }^{3,26-32}$ Similar to previous studies, our results demonstrated that $42 \%$ of carotid revascularizations presented with HD. Patients who underwent CAS had a higher incidence of HD than those who received CEA.

Several limitations of this study should be noted. First, the present study occurred in only one center and was retrospective in nature. Second, a small number of patients were enrolled. Third, we failed to evaluate the site and length of the stenosis, the presence of calcification, the morphology of the plaque, the presence of stenosis on the contralateral side, and the ballooning pressure on the CAS. Fourth, we had no access to data regarding preoperative cardiology evaluation, ${ }^{33}$ HD duration, and clinical events. Fifth, intravenous medication for HD requiring treatment was not administered using identical criteria. Lastly, although guidelines recommend early carotid revascularization, late carotid revascularization was more common in this study. The reason may be due to delayed referral from the primary care physician or early neurological deterioration in the acute stroke phase.

\section{CONCLUSION}

HD can be a challenging event for clinicians to prevent brain hypoperfusion. The present study suggests that early carotid revascularization is as safe as late carotid revascularization with regard to HD. Further large-scale, prospective studies are therefore warranted to determine the correlation between the timing of carotid revascularization and the incidence of HD and clinical events, which is essen- tial for improving CEA and CAS protocols and outcomes.

\section{Acknowledgements}

This work was supported by a grant from the Research Institute of Medical Science, Catholic University of Daegu (2014).

\section{REFERENCES}

1. Brott TG, Hobson RW 2nd, Howard G, Roubin GS, Clark WM, Brooks W, et al. Stenting versus endarterectomy for treatment of carotid-artery stenosis. N Engl / Med 2010;363:1123

2. Bang OY. Intracranial atherosclerosis: current understanding and perspective. / Stroke 2014;16:27-35.

3. Mylonas SN, Moulakakis KG, Antonopoulos CN, Kakisis JD, Liapis CD. Carotid artery stenting-induced hemodynamic instability. J Endovasc Ther 2013;20:48-60.

4. Dangas G, Laird JR Jr, Satler LF, Mehran R, Mintz GS, Larrain $\mathrm{G}$, et al. Postprocedural hypotension after carotid artery stent placement: predictors and short and long term clinical outcomes. Radiology 2000;215:677-83.

5. Howell M, Krajcer Z, Dougherty K, Strickman N, Skolkin M, Toombs B, et al. Correlation of periprocedural systolic blood pressure changes with neurological events in high-risk carotid stent patients. J Endovasc Ther 2002;9:810-6.

6. Rerkasem K, Rothwell PM. Systematic review of the operative risks of carotid endarterectomy for recently symptomatic stenosis in relation to the timing of surgery. Stroke 2009;40:e564-72.

7. Youn SW, Kim HK, Do YR, Do JK, Kwon OC, Lee N, et al. Haemodynamic alterations in cerebral blood vessels after carotid artery revascularisation: quantitative analysis using 2D phase-contrast MRI. Eur Radiol 2013;23:2880-90.

8. Qureshi Al. Acute hypertensive response in patients with stroke: pathophysiology and management. Circulation 2008;118:176-87.

9. Toyoda K, Okada Y, Fujimoto S, Hagiwara N, Nakachi K, Kitazono $\mathrm{T}$, et al. Blood pressure changes during the initial week after different subtypes of ischemic stroke. Stroke 2006;37:2637-9.

10. Sandset EC, Bath PM, Boysen G, Jatuzis D, Kõrv J, Lüders $\mathrm{S}$, et al. The angiotensin-receptor blocker candesartan for 
treatment of acute stroke (SCAST): a randomized, placebocontrolled, double-blind trial. Lancet 2011;377:741-50.

11. Patarroyo SX, Anderson C. Blood pressure lowering in acute phase of stroke: latest evidence and clinical implications. Ther Adv Chronic Dis 2012;3:163-71.

12. Orlandi G, Fanucchi S, Strata G, Pataleo L, Landucci Pellegrini $L$, Prontera $C$, et al. Transient autonomic nervous system dysfunction during hyperacute stroke. Acta Neurol Scand 2000;102:317-21.

13. Meyer S, Strittmatter M, Fischer C, Georg T, Schmitz B. Lateralization in autonomic dysfunction in ischemic stroke involving the insular cortex. Neuroreport 2004;15:357-61.

14. Barron SA, Rogovski Z, Hemli J. Autonomic consequences of cerebral hemisphere infarction. Stroke 1994;25:113-6.

15. Mancia G, Ferrari A, Gregorini L, Parati G, Ferrari MC, Pomidossi $\mathrm{G}$, et al. Control of blood pressure by carotid sinus baroreceptors in human beings. Am / Cardiol 1979;44:895-902.

16. Pilowsky PM, Goodchild AK. Baroreceptor reflex pathways and neurotransmitters: 10 years on. I Hypertens 2002;20:1675-88.

17. Timmers HJ, Wieling W, Karemaker JM, Lenders JW. Denervation of carotid baro- and chemoreceptors in humans. I Physiol 2003;553(Pt 1):3-11.

18. Mangin L, Medigue C, Merle JC, Macquin-Mavier I, Duvaldestin P, Monti A, et al. Cardiac autonomic control during balloon carotid angioplasty and stenting. Can I Physiol Pharmacol 2003;81:944-51.

19. Yakhou L, Constant I, Merle JC, Laude D, Becquemin JP, Duvaldestin $\mathrm{P}$. Noninvasive investigation of autonomic activity after carotid stenting or carotid endarterectomy. I Vase Surg 2006:44:472-9.

20. Lian X, Lin M, Zhu S, Liu W, Li M, Sun W, et al. Risk factors associated with haemodynamic depression during and after carotid artery stenting. / Clin Neurosci 2011;18:1325-8.

21. Cieri E, De Rango P, Maccaroni MR, Spaccatini A, Caso V, Cao $P$. Is haemodynamic depression during carotid stenting a predictor of peri-procedural complications? Eur / Vasc Endovasc Surg 2008;35:399-404.

22. Taha MM, Toma N, Sakaida H, Hori K, Maeda M, Asakura F, et al. Periprocedural hemodynamic instability with carotid angioplasty and stenting. Surg Neurol 2008;70:279-85; discussion 285-6.

23. Leisch F, Kerschner K, Hofmann R, Steinwender C, Grund
M, Bibl D, et al. Carotid sinus reactions during carotid artery stenting: predictors, incidence, and influence on clinical outcome. Catheter Cardiovasc Interv 2003;58:516-23.

24. Nonaka T, Oka S, Miyata K, Mikami T, Koyanagi I, Houkin K, et al. Prediction of prolonged postprocedural hypotension after carotid artery stenting. Neurosurgery 2005;57:472-7.

25. Morgan CE, Lee C), Chin JA, Eskandari MK, Morasch MD, Rodriguez $\mathrm{HE}$, et al. High-risk anatomic variables and plaque characteristics in carotid artery stenting. Vasc Endovascular Surg 2014;48:452-9.

26. Park BD, Divinagracia T, Madej O, McPhelimy C, Piccirillo B, Dahn MS, et al. Predictors of clinically significant postprocedure hypotension after carotid endarterectomy and carotid angioplasty and stenting. I Vasc Surg 2009;50:526-33.

27. Cayne NS, Faries PL, Trocciola SM, Saltzberg SS, Dayal RD, Clair D, et al. Carotid angioplasty and stent-induced bradycardia and hypotension: impact of prophylactic atropine administration and prior carotid endarterectomy. / Vasc Surg 2005;41:956-61.

28. Harrop JS, Sharan AD, Benitez RP, Armonda R, Thomas I, Rosenwasser RH. Prevention of carotid angioplasty-induced bradycardia and hypotension with temporary venous pacemakers. Neurosurgery 2001;49:814-20.

29. Mlekusch W, Schillinger M, Sabeti S, Nachtmann T, Lang W, Ahmadi R, et al. Hypotension and bradycardia after elective carotid stenting: frequency and risk factors. / Endovasc Ther 2003;10:851-9.

30. Pappadà G, Beghi E, Marina R, Agostoni E, Cesana C, Legnani $F$, et al. Hemodynamic instability after extracranial carotid stenting. Acta Neurochir (Wien) 2006;148:639-45.

31. Gupta R, Abou-Chebl A, Bajzer CT, Schumacher HC, Yadav IS. Rate, predictors, and consequences of hemodynamic depression after carotid artery stenting. / Am Coll Cardiol 2006; 47:1538-43.

32. Nano G, Dalainas I, Bianchi P, Stegher S, Bet L, Malacrida $G$, et al. Ballooning-induced bradycardia during carotid stenting in primary stenosis and restenosis. Neuroradiology 2006:48:533-6.

33. Kim JH, Heo SH, Nam HJ, Youn HC, Kim E, Lee JS, et al. Preoperative coronary stenosis is a determinant of early vascular outcome after carotid endarterectomy. / Clin Neurol 2015;11:364-71. 